\title{
L'équipe educativa responsabile e il ruolo formativo del coordinatore pedagogico
}

\section{A equipe pedagógica responsável e o papel formativo do coordenador pedagógico}

\section{The responsible pedagogical team and the training role of pedagogical coordinators}

\author{
Donatella Savio*
}

\begin{abstract}
SOMMARIO
Con riferimento, in particolare, ai servizi educativi per l'infanzia, la capacità di riflettere sulla pratica in modo partecipato viene presentata come la caratteristica peculiare di un'équipe educativa responsabile. Le dinamiche che caratterizzano un'équipe educativa responsabile vengono approfondite facendo riferimento ai concetti di "gruppo di lavoro razionale" (Bion, 1961) e di "identità educativa di gruppo" (Savio, 2011). Vengono quindi delineate le condizioni che favoriscono il funzionamento pedagogicamente responsabile di un'équipe educativa, riprendendo la teoria dei livelli sistemici di Bronfenbrenner (1979) e mettendo in primo piano, per ogni livello sistemico (macro, meso, eso, micro) il ruolo del coordinatore pedagogico. Questa precisazione permetterà di mettere in evidenza il ruolo formativo del coordinatore pedagogico nella misura in cui, sostenendo le dinamiche che attraversano un'équipe educativa responsabile, promuove anche la costruzione di sapere pedagogico, la crescita di consapevolezzacirca l'identità educativa ed il potenziamento dell'intenzionalità professionale, a livello sia individuale che di gruppo.
\end{abstract}

Parole chiave: Capacità riflessiva e partecipativa. Équipe educativa. Servizi educativi per l'infanzia. Coordinatore pedagogico. Gruppo di lavoro razionale.

* Università di Pavia. Dipartimento di Scienze Umanistiche. Pavia, Italia. Piazza Botta n. 6, 27100. E-mail: donatella.savio@unipv.it. 


\title{
RESUMO
}

Especialmente no que diz respeito aos serviços educativos para a infância, a capacidade de refletir sobre a prática de forma participativa é apresentada como uma característica peculiar de uma equipe pedagógica responsável. As dinâmicas que caracterizam uma equipe pedagógica responsável são aprofundadas com base nos conceitos de "grupo de trabalho racional" (Bion, 1961) e "identidade educativa do grupo" (Savio, 2011). São então delineadas as condições que favorecem o funcionamento pedagogicamente responsável de uma equipe educativa, recuperando a teoria dos níveis sistêmicos de Bronfenbrenner (1979) e colocando em primeiro plano, em cada nível do sistema (macro, meso, exo, micro), o papel do coordenador pedagógico. Essa explicação permitirá destacar o papel formativo do coordenador pedagógico, uma vez que, apoiando a dinâmica que atravessa uma equipe pedagógica responsável, sustenta também a construção do saber pedagógico, o crescimento da consciência sobre a identidade educativa e o fortalecimento da intencionalidade profissional, tanto no âmbito individual quanto em grupo.

Palavras-chave: Capacidade reflexiva e participativa. Equipe pedagógica. Serviços educacionais para a infância. Coordenador pedagógico. Grupo de trabalho racional.

\begin{abstract}
With particular reference to educational services for children, the ability to reflect on practice in a participatory way is presented as the peculiar characteristic of a responsible educational team. The dynamics that characterize a responsible educational team are detailed with reference to the concepts of "rational working group" (Bion, 1961) and "educational group identity" (Savio, 2011). The conditions that foster the pedagogically responsible operation of an educational team are therefore defined, taking up the systemic levels of Bronfenbrenner (1979) theory and putting the role of pedagogical coordinator in the foreground, for each system level (macro, meso, eso, micro). This clarification will highlight the training role of the pedagogical coordinator to the extent that, by supporting the dynamics that characterize responsible educational team, it also supports the construction of pedagogical knowledge, the growing awareness about the educational identity and the strengthening of professional intentionality in both individual and group levels.

Keywords: Reflective and participatory capacity. Educational team. Educational services for children. Pedagogical coordinator. "Rational working group".
\end{abstract}




\section{Premessa}

Il documento europeo Proposal for key principles of a Quality Framework for Early Childhood Education and Care ${ }^{l}(2014)$ mette in evidenza come lo sviluppo professionale e le condizioni di lavoro degli operatori educativi siano fattori cruciali per la qualità dei servizi per l'infanzia. Dal documento sembra emergere un profilo della professionalità educativa qualificata che ha tra i suoi tratti peculiari la capacità di riflettere collegialmente sulla pratica, a partire dalla sua osservazione e documentazione, per valutarla e migliorarla co-costruendo un sapere pedagogico condiviso. In sostanza, gli operatori educativi vengono presentati come professionisti riflessivi impegnati in dinamiche partecipative ${ }^{2}$ che affrontano la realtà educativa come un problema da analizzare e risolvere, formulando e mettendo in campo soluzioni idonee, con l'atteggiamento di un ricercatore: infatti, si dice nel documento, il sostegno di una professionalità riflessiva e di pratiche partecipative - con il coinvolgimento anche dei genitori - "rende capace ogni équipe di professionisti di trovare la miglior soluzione per la propria realtà, a partire dall'analisi della situazione sulla base di significati condivisi e di una comprensione comune tanto degli obiettivi del curriculum quanto delle condizioni in cui si opera"'.

Una professionalità vicina a quella descritta da Schön (1983), anche se non in relazione all'ambito educativo, il quale, con riferimento al modello d'indagine di Dewey (1933), descrive un professionista in perenne dialogo riflessivo con la situazione secondo i passi tipici del processo di ricerca: rilevazione del problema imposto dalla realtà, con le sue peculiarità uniche; formulazione di ipotesi di soluzione sulla base delle proprie conoscenze ed esperienze pregresse; messa in campo della soluzione e verifica della sua adeguatezza alle circostanze problematiche specifiche. La proposta di un atteggiamento di questo genere, in

1 Esito di un lavoro realizzato sotto l'egida della Commissione Europea e pubblicato on-line nell'ottobre del 2014, il documento propone e argomenta, sulla base dell'analisi delle politiche dei paesi membri e della letteratura, 10 dichiarazioni di qualità in relazione al sistema dei servizi educativi 0/6, cui si fa riferimento in termini di Early Childhood Education and Care (ECEC) (http://ec.europa.eu/education/policy/strategic-framework/archive/documents/ecec-quality-framework_en.pdf).

2 Nel documento si parla di "partecipatory practices", principalmente in riferimento al coinvolgimento dei genitori nella vita dei servizi educativi; qui e in tutto il contributo richiamo la partecipazione come sinonimo di "lavoro collegiale" e per indicare la caratterizzazione in senso democratico del confronto interno all'équipe educativa.

3 "This enables each team of professionals to find the best solution in their context by analysing the situation on the basis of shared knowledge and understanding of the curriculum's educational goals as well as of the local context", Quality Framework (2014) p. 49 - traduzione di chi scrive. 
rapporto alla professionalità educativa, rende praticabile la libertà di insegnamento e implica un'etica della responsabilità (Bondioli, 2004), per il fatto che richiede all'educatore di orientare il proprio operato, sulla base, non di soluzioni preconfezionate, ma assumendosi in prima persona appunto la responsabilità di leggere i bisogni specifici della realtà specifica, di scegliere oculatamente le soluzioni-risposte ritenute adeguate, in riferimento anche ai propri convincimenti e saperi pedagogici, e di verificarle in modo circostanziato e trasparente.

Inoltre, come precisa il Quality Framework, in ambito educativo, questo modo di procedere deve avvenire in una dimensione collegiale, tale per cui la riflessione sulle proprie pratiche si intreccia con il confronto all'interno dell'équipe. Ciò implica un'équipe che, essa stessa, si definisce in maniera responsabile, cioè che fa proprio l'approccio della ricerca riflessiva all'interno di uno scambio democratico, co-costruendo i propri riferimenti pedagogici convincimenti, valori saperi, pratiche - e arrivando a precisarli e dichiararli, in modo che questi si pongano come la cornice entro cui si modulano la libertà e la responsabilità educative del singolo.

Questa visione complessa della professionalità educativa, in cui si intrecciano capacità riflessiva e partecipativa e che ho deciso di chiamare "responsabile", si apre a una molteplicità di approfondimenti relativi ad ambiti di ricerca vicini, ma non sempre intrecciati: da una parte, la ricca e recente letteratura sia sulla professionalità riflessiva di chi opera in educazione sia sui processi partecipativi di co-costruzione della conoscenza, dall'altra, l'ampio, differenziato e consolidato dibattito sulle dinamiche del gruppo di lavoro ${ }^{4}$. Su questo sfondo, con il mio contributo, vorrei affrontare il tema della professionalità dell'équipe responsabile, mantenendomi all'interno di un quadro teorico circoscritto, per mettere a fuoco sia gli aspetti che possono svilupparsi quando la professionalità riflessiva viene realizzata all'interno di un gruppo di lavoro educativo, sia le modalità attraver-

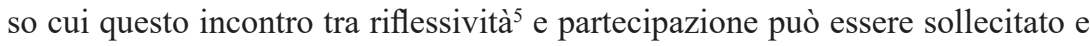

4 La letteratura in questi ambiti è davvero molto vasta e diffusa a livello internazionale, tanto che risulta difficile fornire dei riferimenti esaustivi. Per una rassegna sul pensiero riflessivo in rapporto alla professionalità docente cfr. Montalbetti (2005). Rispetto alle dinamiche partecipative, i riferimenti di fondo sono da una parte il paradigma del costruttivismo sociale, dall'altra il pensiero di Freire (1970), il suo accento sui processi democratici di costruzione della conoscenza e sul loro valore emancipativo; in ambito educativo, queste prospettive sono state affrontate, in particolare da Dahlberg, Moss, Pence (1999) e da Moss, Dahlberg (2008), con riferimento al tema della qualità dei servizi per l'infanzia. Nel panorama italiano, a partire dagli anni '80, si è sviluppato un filone di studi che affronta la dimensione della riflessività e della partecipazione in rapporto ai processi di valutazione della qualità dei contesti educativi per la prima infanzia; una presentazione di questo filone nei suoi sviluppi più recenti si trova in Bondioli, Savio (2015).

5 Parlerò di riflessività sempre nel senso in cui Dewey e Schön l'intendono, e cioè come a un modo di relazionarsi alla pratica secondo i processi tipici dell'indagine; a tal proposito, Mon- 
sostenuto. In particolare discuterò i tratti dell'équipe educativa responsabile, mettendoli in relazione con il concetto di gruppo di "lavoro razionale" di Bion (1961), un riferimento fondamentale per la teoria dei gruppi in ambito psicoanalitico, e con quello di "identità educativa", da me precisato in un precedente lavoro (Savio, 2011). Inoltre, riprenderò la teoria ecologica di Bronfenbrenner (1979) per delineare le condizioni che consentono il funzionamento di un'équipe educativa responsabile, riferendomi, in particolare, al ruolo del coordinatore pedagogico, che, in questa prospettiva, assume una funzione formativa ${ }^{6}$ : formativa in quanto, come dirò meglio più avanti, sostenere nell'équipe un rapporto partecipato e riflessivo con la pratica determina, oltre alla co-costruzione di sapere pedagogico, una crescita di consapevolezza rispetto ai riferimenti pedagogici comuni e dunque un potenziamento dell'intenzionalità professionale.

\section{L'équipe educativa responsabile come "gruppo di lavoro razionale"}

Descrivere un'équipe educativa responsabile come un gruppo che affronta il proprio compito in modo riflessivo e partecipato permette di sviluppare una

talbetti (2005) mette in evidenza che questa coincidenza tra pensiero riflessivo e ricerca è tipica della professionalità dell'insegnante in quanto consiste nel progettare e verificare interventi che mirano a modificare in senso evolutivo la realtà, cioè i discenti, sulla base dell'osservazione dei loro bisogni e dei propri riferimenti pedagogici.

6 In questo contributo si considererà la figura del coordinatore pedagogico esclusivamente in relazione al suo possibile ruolo rispetto a un'équipe educativa che si caratterizzi come responsabile nel senso detto. Occorre precisare però che, con riferimento, in particolare, alla realtà italiana, questa figura svolge molteplici funzioni. Nasce negli anni ' 70 con compiti, soprattutto relativi all'équipe educativa di un servizio per l'infanzia, quali il supporto pedagogico, l'organizzazione del lavoro, la promozione della formazione, la cura dei rapporti con le famiglie e con l'esterno - l'amministrazione, l'utenza, gli altri nidi -. Con il diffondersi dei servizi integrativi (centri gioco, spazi per le famiglie ecc.) - a partire dagli anni '80 - e privati o convenzionati - a partire dagli anni '90-, il coordinatore pedagogico si trova sempre più a svolgere anche funzioni di governo del sistema, che implicano la rilevazione dei bisogni educativi del territorio, l'individuazione e la gestione delle risorse, il monitoraggio del sistema dei servizi, la salvaguardia ed il controllo della qualità. Benché in Italia le leggi regionali sui servizi educativi per l'infanzia prevedano la presenza di un coordinamento pedagogico, questa presenza risulta non omogenea sul territorio nazionale: è più diffusa e radicata al centro-nord, dove, nei comuni più grandi e a livello provinciale, esistono reti ed équipe di coordinamento con funzioni di raccordo e di promozione della coerenza educativa tra i servizi educativi per l'infanzia del territorio di riferimento. Una situazione particolarmente articolata è presente nella Regione Emilia Romagna, nella quale, dal 2004, sono stati istituiti i coordinamenti pedagogici provinciali (CCP), a loro volta coordinati e monitorati a livello regionale. Per approfondire questi punti cfr. Bondioli, 2002; Musatti, Mayer 2003; Benedetti, 2009; Catarsi, 2010; Silva, 2015. 
connessione con il concetto bioniano di "gruppo di lavoro razionale" (Bion, 1961). Senza entrare nei dettagli della teoria bioniana dei gruppi, vediamo in che termini può essere sviluppata tale connessione.

Secondo Bion, quando un insieme di individui opera congiuntamente in vista di un compito comune, costituisce un gruppo che si dota di una struttura razionale. La razionalità del gruppo si esprime nella capacità dei suoi membri di cooperare, di darsi un'organizzazione, di produrre attività finalizzate all'esecuzione del compito e, quindi, di mantenersi su un piano di realtà, dotandosi a tale scopo di metodi "razionali e pertanto, sia pure in forma embrionale, scientifici" (Bion, 1961, p. 153). Soprattutto, il gruppo di lavoro razionale è caratterizzato da alcune idee preminenti: la consapevolezza della validità di "un approccio razionale o scientifico ai problemi”" (Bion, 1961, p. 107); la convinzione che un processo di sviluppo è sempre necessario, che non si dà una capacità acquisita per istinto e che l'apprendimento avviene attraverso l'esperienza. In altre parole, il gruppo di lavoro razionale è attraversato dalla convinzione che si può apprendere come affrontare il compito solo attraverso l'esperienza, cioè solo aprendosi al confronto diretto con la realtà e sviluppando tale confronto secondo un atteggiamento "scientifico" e razionale: un confronto quindi che, sembradi poter dire, si caratterizza come un problema da risolvere e si realizza come un processo di indagine.

Mi pare che l'équipe educativa responsabile, così come è stata definita, rispecchi pienamente le caratteristiche del gruppo di lavoro razionale. Innanzitutto perché condivide un compito comune, che nello specifico ha un carattere pedagogico; un compito oltretutto fortemente radicato nella realtà, quella concreta del particolare contesto educativo in cui i membri del gruppo operano. La possibilità che questo compito venga svolto in maniera positiva, cioè producendo un "buon" ambiente educativo per i bambini che lo vivono, richiede che i membri dell'équipe cooperino. La cooperazione produce capacità di organizzarsi per raggiungere gli obiettivi condivisi, stabilendo chi fa che cosa e distribuendo equamente pesi e responsabilità, ma riguarda anche il piano molto più complesso dell'apprendere insieme dall'esperienza, dotandosi di metodi "razionali" e "scientifici", per quanto in forma rudimentale, così come li definisce Bion. Su questo piano, lo abbiamo visto, l'équipe educativa responsabile rifiuta soluzioni preconfezionate e invece, in modo partecipato, apre un dialogo riflessivo - o pseudo-scientifico secondo la prospettiva bioniana - con la specifica realtà di riferimento e perciò ritmato dai processi tipici dell'indagine: la osserva, osserva i bambini concreti con cui opera, per definire precisamente problemi ed esigenze, quindi, sulla base dei propri valori educativi e delle proprie conoscenze pregresse, formula, mette in atto e verifica ipotesi di soluzione ad hoc. 
Dunque, in questa cornice, la "razionalità" cui si riferisce Bion può essere interpretata come una sorta di riflessività deweyana declinata in termini di gruppo. Procedendo in questo modo, l'équipe apprende dall'esperienza, co-costruisce cioè un sapere pedagogico sempre rinnovato dalle sollecitazioni uniche proposte dalla pratica, sapere che davvero incide sulla professionalità di tutti i membri del gruppo arricchendola e trasformandola.

Come ho già suggerito, si tratta di un modo di procedere complesso. Innanzitutto perché richiede di aprirsi al rischio dell'indeterminato e quindi, di assumersi la responsabilità degli esiti imprevedibili che verranno in seguito al dialogo riflessivo con la realtà, e di farlo in modo continuamente rinnovato, mai finito una volta per tutte. Ma anche perché, come già evidenziato, è un procedere che va realizzato in modo partecipato, cooperando. Il pensiero di Bion (1961) aiuta a comprendere le caratteristiche di tale complessità. La struttura razionale del gruppo è psicologicamente forte, ma si trova in lotta con tendenze emotive che premono verso obiettivi molto diversi da quelli esplicitamente assunti dal gruppo stesso. Queste tendenze emotive hanno a che fare sostanzialmente con due aspetti. Da una parte con la contraddizione che l'individuo sperimenta quando fa esperienza del gruppo: ha bisogno della dimensione sociale, ma in essa contemporaneamente vede frustrati i desideri connessi alla sua esistenza come individuo (Bion, 1961, p. 61-62). D'altra parte preme, sempre a livello individuale, la difficoltà a fare i conti con le fatiche della crescita, e, quindi, il desiderio di uno stato in cui, per istinto o magicamente, si è subito cresciuti, capaci e attrezzati per la vita di gruppo (Bion, 1961, p. 97-99).

Credo che tali tendenze, che entrano in contrasto con la struttura razionale del gruppo, possano trovare una declinazione particolare in relazione alla vita di un'équipe educativa responsabile. Riguardo alla dinamica che vede l'individuo nel gruppo contemporaneamente bisognoso di questa socialità e frustrato nell'espressione della sua individualità, mi pare che possa essere tradotta come la difficoltà per i singoli educatori di vedere il proprio punto di vista educativo individuale perdersi nell'anonimato e la propria libertà di insegnamento limitata, se accettano di contribuire e di aderire all'équipe in quanto unità pedagogica con un proprio profilo pedagogico che comprende e supera quelli individuali. Questa difficoltà potrebbe intralciare i processi di cooperazione, cioè partecipativi, che sono l'ossatura di ogni gruppo di lavoro e dell'équipe educativa responsabile in particolare, sviluppando una resistenza alla co-costruzione di riferimenti pedagogici condivisi per salvaguardare viceversa l'esistenza delle prospettive dei singoli. Nello stesso senso, potrebbe agire la difficoltà ad accettare le fatiche connesse all'apprendere dall'esperienza, non solo relative al confronto riflessivo con la realtà educativa che sollecita costantemente nuovi percorsi di apprendimento, ma anche al lavorare in gruppo secondo le modalità 
del confronto democratico. Si tratta di imparare ad argomentare il proprio punto di vista, ad ascoltare i punti di vista di ognuno e, soprattutto, a confrontarsi con l'obiettivo di co-costruire riferimenti educativi condivisi, propri dell'équipe intesa appunto come unità pedagogica. Anche il desiderio di scavalcare le fatiche che questo processo di apprendimento porta con sé, come a quello già accennato di non perdere la propria prospettiva educativa in quella del gruppo, potrebbe lavorare contro la realizzazione delle dinamiche partecipative, che rappresentano, insieme all'approccio razionale e riflessivo all'agire educativo, una delle cifre dell'équipe educativa responsabile. Considererò più avanti quali condizioni favoriscono il contenimento di tali tendenze a sostegno del funzionamento responsabile dell'équipe educativa.

\section{L'équipe educativa responsabile come identità educativa}

Un'équipe educativa responsabile, come ho già detto, co-costruisce dei riferimenti pedagogici di gruppo che comprendono, ma in qualche misura superano, quelli dei suoi membri. Per chiarire la natura di questi riferimenti e dei processi che contribuiscono a costruirli è utile rifarsi alle considerazioni sul concetto di identità educativa che ho proposto in un precedente lavoro (Savio, 2011), distinguendo tra identità educativa del singolo e del gruppo.

In termini generali, l'identità educativa può essere intesa come l'intreccio tra il piano delle idee pedagogiche - che possono comprendere i valori, i convincimenti, le teorie, le conoscenze costruite con l'esperienza - e quello delle azioni che ne derivano. È possibile parlare su queste basi di identità educative individuali, dei singoli operatori, e di identità educativa di gruppo, dove, in questo secondo caso, si fa riferimento a ciò che risulta dall'intreccio e dalle consonanze tra le identità educative degli educatori che fanno parte di quel gruppo.

Se si considera la possibilità che il piano delle idee pedagogiche abbia dei lati oscuri, e cioè che non tutti i riferimenti dell'educatore siano a lui presenti in maniera chiara ed esplicita, l'intreccio delle dimensioni che determinano l'identità educativa si fa più complesso. Essa comprenderebbe anche le pedagogie latenti (Becchi, 2005; Bondioli, 2002), cioè i riferimenti presenti in maniera non del tutto esplicita nella mente dell'educatore e che tuttavia guidano le sue azioni in modo sotterraneo ma molto potente, magari anche non del tutto coerente con quanto richiesto dai riferimenti espliciti. Potrebbe perciò darsi il caso di un educatore che, sulla base del valore democratico del libero confronto tra punti di vista, dichiara di voler sostenere l'autonomia dei bambini negli 
scambi di contrattazione sociale (ad es. nelle discussioni riguardo a chi fa la mamma ed a chi fa il papà in una situazione di gioco simbolico), ma che agisce in modo da proporsi sempre come mediatore di questi scambi sulla base del convincimento implicito che in età prescolare non vi è la capacità di sostenerli. A livello di identità di gruppo, gli intrecci e le aree di sovrapposizione tra le identità individuali potrebbero riguardare tanto le pedagogie esplicite che quelle latenti, con il risultato possibile di un'équipe che abbraccia in modo dichiarato e condiviso certi convincimenti, ma che ne pratica altri.

Contro questa possibilità, il lavoro di un'équipe educativa responsabile, proprio in virtù del suo modo di procedere riflessivo e partecipato, sollecita processi di esplicitazione e quindi di costruzione di riferimenti consapevoli, tanto a livello di identità educativa individuale che di gruppo.

A livello di identità educativa individuale, si può dire che già l'affrontare da soli in modo riflessivo i problemi posti dalla realtà educativa in cui si opera "stana" gli impliciti, mette a fuoco "cosa si fa effettivamente" e di conseguenza impone di chiedersi "perché lo si fa", quale idea - valore, convincimento, teoria - orienta quel fare: l'educatore dell'esempio precedente, se assumerà una postura riflessiva, vedrà la sua tendenza a mediare le contrattazioni tra bambini e sarà "costretto" a dichiarare il convincimento che lo guida.

Questo processo però è decisamente obbligato e più stringente se avviene in modo partecipato, cioè all'interno di un'équipe che abbraccia l'approccio riflessivo e, come direbbe Bion, fa leva sulla propria struttura razionale; infatti se il singolo può cedere alla tentazione di distogliere lo sguardo e non vedere le sue pedagogie implicite, sarà l'équipe a proporre lo svelamento e quindi la presa di consapevolezza del profilo dell'identità educativa individuale. Non solo. Il confronto tra i singoli all'interno dell'équipe spinge ancora più in profondità questo processo. Ognuno è sollecitato a chiarire "come fa" e "perché fa quello che fa", e questa sollecitazione costringe a prendere consapevolezza dei propri riferimenti impliciti e ad argomentarli, ma anche a considerare se mantenerli o rigettarli, con un atto di assunzione di responsabilità ancora individuale. Naturalmente va considerato che questo processo di slatentizzazione pedagogica è sempre parziale e mai finito, perciò richiede di essere continuamente sostenuto e rinnovato.

A livello di identità educativa di équipe, questo processo produce uno sviluppo analogo. Infatti, nella presa di consapevolezza e ridefinizione del profilo dell'identità educativa dei singoli, si produce una conoscenza nuova - perché prima sconosciuta ma anche perché sapendola la si rielabora - che è il passo preliminare per la costruzione di un sapere analogo a livello di identità educativa dell'équipe. In quanto membri di un'équipe, i singoli hanno la responsabilità di condividere riferimenti e pratiche, dunque di co-costruire un'identità educativa 
comune, perché tale condivisione garantisce coerenza pedagogica tra le esperienze e relazioni proposte ai bambini. Il confronto tra le identità educative dei singoli, via via consapevolmente ridefinite in rapporto anche, non va dimenticato, al dialogo riflessivo con la pratica educativa, è perciò finalizzato a trovare accordi, a negoziare, chiarire e scegliere responsabilmente in modo esplicito quali sono i riferimenti pedagogici condivisi, cioè a chiarire e scegliere, passando dal singolare al plurale e dalla constatazione all'intenzione, "perché e come vogliamo fare quello che facciamo". Dunque, il sapere co-costruito all'interno di un'équipe responsabile consiste nella rielaborazione partecipata dell'identità educativa condivisa. Anche in questo caso è un processo mai finito, perché dalla realtà educativa emergono sempre nuove sfide che sollecitano le identità educative individuali e quindi quella di gruppo a rinnovate consapevolezze e co-costruzioni di significato.

È del tutto evidente che, sia a livello del singolo educatore che dell'équipe, il processo delineato ha importanti ricadute formative, in termini appunto sia di accrescimento di consapevolezza professionale sia di elaborazione di sapere pedagogico. Un processo che può strutturarsi come formazione in servizio a carattere permanete se si danno condizioni favorevoli al suo rinnovarsi e consolidarsi. Nel prossimo paragrafo considererò alcune di queste condizioni.

\section{Le condizioni per un'équipe educativa responsabile: il ruolo formativo del coordinatore pedagogico}

Per precisare le condizioni che favoriscono - nei servizi rivolti all'infanzia in particolare - il funzionamento responsabile di un'équipe educativa ed il ruolo cruciale che svolge il coordinatore pedagogico in tal senso, è utile il riferimento alla teoria ecologica di Bronfenbrenner (1986). Secondo questa prospettiva la qualità dell'ambiente di cui l'individuo in via di sviluppo ha esperienza diretta, il microsistema, dipende dalle interconnessioni tra quest'ambiente e situazioni ambientali che si collocano concentricamente a livelli diversi.

L'ambiente è quindi concepito come una sorta di sistema di sistemi inseriti uno nell'altro. In particolare, Bronfenbrenner individua quattro livelli ambientali: oltre al microsistema, parla di mesosistema, ecosistema, macrosistema. Se il microsistema è il livello ambientale esperito direttamente dall'individuo in evoluzione (ad es., per il bambino, la scuola dell'infanzia o la famiglia), il mesosistema riguarda le relazioni tra microsistemi (ad es. tra le insegnanti della scuola dell'infanzia e i genitori del bambino). L'esosistema comprende situazioni 
ambientali cui l'individuo in via di sviluppo non partecipa direttamente, ma che nondimeno hanno una qualche influenza sui microsistemi (ad es., per il bambino che frequenta una scuola dell'infanzia, le attività dell'équipe educativa, i suoi rapporti con il coordinatore pedagogico). Infine, il macrosistema è il livello del sistema di credenze, che determina trasversalmente le congruenze dei livelli inferiori sia nei contenuti che nella forma (ad es. la cultura della partecipazione presente in un certo momento storico di una certa società, determinerà sia politiche educative aperte al confronto sia rapporti democratici all'interno dell'équipe educativa, tra insegnanti e genitori, tra insegnanti e bambini ecc).

Con questi riferimenti, mi pare che le condizioni a favore del funzionamento responsabile di un'équipe educativa possano essere indicate per ogni livello ambientale; metterò però in evidenza, in particolare, come si realizzano soprattutto a livello di esosistema nel rapporto tra équipe e coordinatore pedagogico.

Livello macrosistemico. Per quanto riguarda il livello del macrosistema, mi preme brevemente sottolineare come un orientamento culturale e politico che riconosca il valore delle pratiche riflessive e partecipative nella professione educativa sia un requisito fondamentale per il realizzarsi di un'équipe educativa responsabile. Lo stesso Quality Framework (2014), il già citato documento europeo (cfr. nota 1), indica come elemento cruciale per la qualità educativa la presenza di politiche che a livello nazionale, regionale e locale sostengano la professionalizzazione continua degli educatori, secondo modalità di ricerca riflessiva e partecipata. Un ponte cruciale tra le politiche educative e la realtà dei servizi concreti si realizza attraverso il ruolo del coordinatore pedagogico. Infatti, se il piano politico afferma un certo tipo di professionalità educativa fornendo gli orientamenti e le risorse per realizzarla, è il coordinatore che può sostenere il nutrimento culturale di una certa équipe educativa nella direzione auspicata, attraverso la messa a disposizione di informazioni, formazioni, strumenti metodologici e teorici per la professionalizzazione in senso riflessivo e partecipato.

Livello esosistemico. La funzione fondamentale del coordinatore, tuttavia, si sviluppa a livello di esosistema, cioè delle sue relazioni con l'équipe che si propone di funzionare in maniera responsabile. Facendo riferimento a quanto argomentato a proposito delle dinamiche complesse che caratterizzano un'équipe educativa responsabile, mi pare che il coordinatore possa sostenerle, esercitando sostanzialmente due funzioni, tra loro intrecciate, ma qui distinte per esigenze di analisi: di contenimento emotivo e di facilitazione del confronto democratico.

Riguardo alla funzione di contenimento emotivo, occorre rifarsi ancora alla teoria bioniana. In estrema sintesi, secondo questa prospettiva l'apprendimento è reso possibile dall'incontro con ciò che non si conosce e dalla capacità di tollerare il dolore mentale che questo incontro comporta: paura della confusione, caos di fronte a esperienze non ancora classificate, impotenza di fronte al non 
sapere, timore di essere inadeguati (Salzberger-Wittenberg, Henry-Polacco \& Osborne, 1987). Se questo dolore può essere "contenuto", cioè accolto, nominato e quindi reso pensabile, allora il processo di apprendimento può dispiegarsi; si tratta di una funzione di contenimento che viene in origine attivata dalla madre, permettendo al bambino di sperimentarla e quindi di introiettarla come "mente per pensare i pensieri".

Sono dinamiche che riguardano da vicino il rapporto tra coordinatore e un'équipe educativa che apre un dialogo riflessivo con la realtà operativa. Per quest'ultima aprire un tale dialogo significa abbandonare soluzioni preconfezionate per stare a contatto con i vissuti dolorosi connessi all'apprendere dall'esperienza, con l'indeterminatezza del problema unico proposto da tale realtà e con l'incertezza degli esiti che il percorso di indagine riflessiva produrrà. Sono vissuti che possono intralciare il funzionamento razionale dell'équipe esponendola al rischio di trovare soluzioni frettolose o ripiegare su strategie preconfezionate.

Se si considera poi che la complessità della vita del gruppo attiva fenomeni regressivi (Bion, 1961, p. 151), depotenziando le capacità di contenimento proprie dei suoi membri, risulta evidente quanto possa essere rilevante che la funzione di contenimento di tali vissuti venga svolta dal coordinatore. In quanto figura in stretta relazione con l'équipe, ma anche esterna ad esso, può svolgere un ruolo sia di cura del benessere mentale sia di mediazione con il mondo "là fuori", con il piano della realtà; dunque, proprio come la madre, può attivare la propria capacità di cogliere e pensare i vissuti che intralciano l'équipe nel suo approccio riflessivo al compito, permettendo il dispiegarsi dei processi di apprendimento dall'esperienza.

Per il coordinatore che voglia muoversi in questa direzione, si tratta concretamente di assumere un preciso stile relazionale, che prevede alcuni passi. In primo luogo, occorre che rinunci ad esercitare una posizione di potere, di leadership direttiva, controllante, sanzionatoria, posizione che può facilmente essere attivata come risposta difensiva alla complessità del ruolo. Occorre viceversa che si attenga a un ruolo, appunto, di coordinamento, che si sostanzia nell'affiancare e sostenere attivamente la vitalità e il benessere del gruppo di lavoro, cogliendo le sue difficoltà a sostare nell'incertezza, nel non sapere cosa dovrà affrontare, come lo affronterà e se riuscirà e imparerà ad affrontarlo, per aiutarlo a perseverare nella prospettiva secondo cui "il percorso si fa mentre si fa". Questo aiuto si realizza non tanto nel verbalizzare al gruppo tali difficoltà per approfondirle - compito più appropriato per un esperto chiamato ad affrontare le dinamiche di gruppo -, quanto nel riconoscerle, quindi poterle pensare, e sostenerne il superamento, richiamando gli aspetti propri del gruppo razionale: mantenendo la "barra dritta" verso il compito, cioè verso il mettere a punto e 
alla prova proposte educative coerenti con i propri riferimenti pedagogici e rispondenti alle esigenze sollevate dal contesto particolare; contribuendo a rafforzare l'organizzazione del gruppo in tal senso, cioè concretamente, ad esempio, programmando tempi (di incontro, per l'osservazione ecc.) e strumenti (per l'osservazione, per l'analisi dell'osservazione, per la formulazione di soluzioni educative nuove ecc.) dedicati al compito riflessivo comune; promuovendo le dinamiche di cooperazione, ad esempio, invitando a definire i diversi ruoli, "chi fa che cosa" nella realizzazione del compito riflessivo comune. In sostanza, per dirlo con Bion, il coordinatore dovrebbe porsi come un capo innocuo, privo di influenza sul gruppo, ma attivo nel mantenere il contatto con la realtà esterna (Bion, 1961, p. 188).

Un'analoga funzione di contenimento può essere svolta dal coordinatore in relazione alle tendenze emotive che nel gruppo interferiscono con il processo partecipativo, di confronto democratico teso a negoziare significati condivisi. Su questo piano le difficoltà riguardano, lo ricordo, sia la messa in secondo piano della propria individualità sia le fatiche dell'imparare a stare dentro le dinamiche partecipative. Anche in questo caso il coordinatore, proprio in funzione del suo essere contemporaneamente in relazione col gruppo, ma non parte di esso, può più facilmente dei membri dell'équipe vedere tali difficoltà e contenerle, puntando sul rafforzamento della sua struttura razionale e cioè, vale la pena di ribadirlo, sul riferimento al compito comune con l'organizzazione di attività e ruoli che esso richiede.

Non solo. Il coordinatore può fare un passo ulteriore per promuovere la dimensione della cooperazione interna al gruppo di lavoro razionale, sempre in virtù della sua posizione di vicinanza esterna al gruppo, esercitando in prima persona una funzione di facilitazione del confronto democratico. In questo senso può essere utile il riferimento allo stile relazionale di "promozione dall'interno" messo a punto da me e da Bondioli in percorsi di valutazione di contesto formativa e partecipata, rivolti prevalentemente ad équipe di educatori di servizi per l'infanzia (Savio, 2013; Bondioli \& Savio, 2013, 2015). Anche in questi percorsi si propone agli educatori un dialogo riflessivo partecipato con la realtà in cui operano, partendo dalla sua valutazione per arrivare a condividerne il profilo di qualità, i punti forti e le fragilità che la caratterizzano, e quindi a definire consensualmente come intervenire per migliorarla, dopo aver concordato "perché e come si vorrebbe fare quello che si fa"; a tale scopo è previsto l'accompagnamento di un facilitatore, il cui ruolo è stato precisato attraverso diverse esperienze di ricerca formazione. In relazione, in particolare, al sostegno del confronto democratico, e dunque alla funzione che stiamo approfondendo in relazione al coordinatore di un'équipe educativa responsabile, il ruolo del 
facilitatore che "promuove dall'interno" può essere in sintesi caratterizzato come segue:

- è una attore coinvolto, partecipa attivamente al compito;

- ha un atteggiamento neutrale ma aperto e curioso verso qualsiasi punto di vista espresso;

- promuove processi di esplicitazione ed ascolto dei punti di vista di tutti i membri del gruppo;

- stimola il confronto finalizzato alla negoziazione e la condivisione di punti di vista sulla realtà di riferimento.

In termini più specifici, si può dire che attiva funzioni analoghe a quelle del tutoring (Wood, Bruner, \& Ross, 1976), interpretate in relazione a una dimensione di gruppo e ad un compito aperto: dal punto di vista affettivo, promuove e monitora il coinvolgimento dei partecipanti nel compito, ma contiene anche la frustrazione connessa alla scoperta di punti di vista discordanti dal proprio; dal punto di vista socio-cognitivo, mantiene la direzione verso l'esecuzione del compito riflessivo concordato, e, per farlo, favorisce l'esplicitazione di idee/possibilità solo intuite e accennate dai partecipanti, portando tra queste in evidenza quelle tra loro compatibili, per sostenere la negoziazione di punti di vista condivisi nel gruppo.

Questi riferimenti mettono in evidenza la complessità del ruolo del coordinatore nel suo rapporto di sostegno all'équipe educativa responsabile, un sostegno che si può ben indicare come azione formativa in quanto determina acquisizione di consapevolezza educativa, elaborazione di sapere pedagogico e capacità di cooperazione, a livello sia del singolo educatore che del gruppo. In questo senso, il coordinatore pedagogico rappresenterebbe una risorsa preziosa e soprattutto sostenibile per la formazione permanente in servizio di un'équipe educativa nella direzione auspicata, come abbiamo visto, dal Quality Framework (2014).

Una risorsa che però non si esaurisce a livello esosistemico e viceversa, si sviluppa anche a livello meso e micro sistemico. Brevemente, poiché come anticipato il fuoco del mio approfondimento riguarda il livello esosistemico, accennerò a quelli che mi sembrano essere alcuni tra questi sviluppi.

Livello mesosistemico. A livello mesosistemico, il ruolo del coordinatore riguarda la sua funzione di ponte tra l'équipe/le équipes con cui opera e le altre agenzie educative per l'infanzia collocate sul territorio, prime fra tutte le famiglie. Nello svolgere questa funzione a vari livelli, il coordinatore può fornire un sostegno al funzionamento responsabile dell'équipe sollecitandola a definire strategie per presentarsi come tale agli interlocutori territoriali. Si tratterebbe, per il coordinatore, di promuovere la produzione di una documentazione (opuscoli, materiale multimediale, ecc.) che comunichi l'identità educativa dell'équipe nei 
suoi valori, convincimenti e pratiche di riferimento, e dunque anche nel suo modo di essere costantemente aperta al dialogo riflessivo con la realtà e al confronto democratico. Nel sollecitare la "comunicazione di sé" da parte dell'équipe, il coordinatore svolge una duplice funzione, a favore sia della definizione sia dell'arricchimento dell'identità educativa del gruppo. Da una parte perché, nel predisporre un materiale di questo tipo, l'équipe ha modo di "guardarsi da fuori”, dunque di acquisire una consapevolezza più solida della propria identità educativa, che a sua volta ne alimenta ulteriormente l'elaborazione e definizione partecipata. D'altra parte perché, se tra le strategie per la "comunicazione di sé" il coordinatore promuove anche il confronto aperto e democratico con le altre agenzie educative, l'équipe sarà sollecitata a considerare altre prospettive e identità aprendo la propria a ulteriori elaborazioni e più ampie partecipazioni. Tutto questo rafforza anche la dimensione dell'assunzione di responsabilità, in quanto dichiarare i propri riferimenti educativi in modo chiaro ed esplicito, ma anche aperto al dialogo, significa appunto porsi nella condizione di rispondere in prima persona delle proprie dichiarazioni ed azioni argomentandone le ragioni.

Livello microsistemico. Per quanto concerne invece il livello microsistemico, il ruolo del coordinatore a sostegno del funzionamento responsabile dell'équipe educativa può concretizzarsi nel mettersi a disposizione come strumento per rilevare ciò che emerge nei momenti di più stretto dialogo con la pratica. In altre parole, proprio in qualità di attore vicino, ma non immerso nella realtà educativa del rapporto con i bambini, può affiancare il lavoro degli educatori per osservare quali sono le sollecitazioni emergenti nella relazione con tale realtà e come questa risponde alle soluzioni, elaborate in modo riflessivo e partecipato, messe in campo per risolverli. Un'osservazione che condurrà con strumenti diversi a seconda delle diverse esigenze e sulla base di quanto concordato all'interno dell'équipe: carta e penna, videoregistrazioni, strumenti di valutazione, ecc. Questa possibilità, oltre sollevare gli educatori dall'onere di realizzare in prima persona le rilevazioni, permette di facilitare il dispiegamento dei processi riflessivi per due ragioni. In primo luogo, perché evita rilevazioni che rispondano all'esigenza dell'educatore di mostrare la realtà come vorrebbe più che come la percepisce, e dunque permette di avere materiale descrittivo sufficientemente attendibile, ma anche perché lo sguardo del coordinatore, in quanto esterno però vicino, può cogliere aspetti che gli sguardi interni degli educatori non riescono a percepire, riuscendo contemporaneamente a collocare quanto rileva nell'orizzonte di senso proprio e peculiare della realtà osservata perché lo conosce. 


\section{Alcune osservazioni conclusive}

Per come è stato fin qui delineato, il ruolo del coordinatore pedagogico non è banale e richiede una professionalità alta e specializzata. Si apre quindi la questione della sua formazione, che evidentemente non può limitarsi all'acquisizione di contenuti psico-pedagogici, ma che deve prevedere percorsi di addestramento alla riflessione partecipata sulla pratica educativa su due livelli: sul livello della comprensione delle dinamiche che caratterizzano un'équipe educativa responsabile, ma anche su quello delle strategie con cui è possibile sostenerle da parte di un ruolo di coordinamento. Inoltre, mi pare che l'esercizio di una professionalità così complessa richieda essa stessa un supporto continuo in termini di supervisione, che potrebbe essere anche realizzata tra pari. Sto pensando a reti di coordinatori che vicendevolmente si supportano nel riflettere insieme sulle provocazioni uniche a cui devono far fronte nel rapporto con le rispettive équipe responsabili, praticando quello stesso atteggiamento di dialogo riflessivo con la realtà professionale, in una dimensione di confronto democratico, che mirano a sostenere in quelle équipe $e^{7}$. Insomma si tratta di formare e sostenere nel tempo coordinatori doppiamente "responsabili", visto che fanno parte di équipe educative responsabili per sostenere la loro capacità di promuovere équipe educative responsabili. È evidente che una formazione e un sostegno di questo genere chiamano in causa, circolarmente, il livello macrosistemico, e cioè devono essere previste a livello di politiche educative non solo locali e regionali, ma anche nazionali, con un'assunzione di responsabilità questa volta tutta politica.

\section{TESTI CITATI}

Becchi, E. (2005). Pedagogie latenti: una nota. Quaderni della didattica della scrittura, 3, p. 105-113.

Benedetti, S. (2009). I coordinamenti pedagogici nella regione Emilia Romagna: un investimento sulla professionalità. Infanzia, 3, maggio-giugno 2009, pp. 182-185.

7 A questo proposito ricordo che in alcune realtà territoriali italiane queste reti di coordinamento già esistono (cfr. nota 6); in particolare, la rete dei Coordinamenti Pedagogici provinciali della Regione Emilia Romagna ha realizzato un percorso per molti versi analogo alla supervisione riflessiva partecipata tra pari cui si fa qui riferimento (cfr. Gariboldi, 2015). 
Bion, W. R. (1961). Esperienze nei gruppi. Trad. it. Roma, Armando Armando, 1971.

Bondioli, A. (2000). Riflettere sulla giornata scolastica: uno strumento e un percorso di valutazione formativa. In: Bondioli A., Ferrari M. (2000) (a cura di). Manuale di valutazione del contesto educativo: teorie, modelli, studi per la rilevazione della qualità della scuola, Milano, Franco Angeli, pp. 345-369.

Bondioli, A. (2002). Il coordinatore pedagogico: una figura chiave per la qualità dei servizi per l'infanzia. In: Bondioli A. (a cura di). La qualità negoziata. Il progetto pedagogico del nido e la sua valutazione, Azzano S. Paolo (BG), Edizioni Junior, p. 89-105.

Bondioli, A. (2004). Promuovere dall'interno. In: Bondioli A., Ferrari M. (a cura di). Educare la professionalità degli operatori per l'infanzia, Azzano S. Paolo (BG), Junior, pp. 61-76.

Bondioli, A., \& Savio D. (a cura di) (2013). Participação e qualidade em educação da infância, Curitiba: Editora UFPR.

Bondioli A., \& Savio D. (a cura di) (2015). La valutazione di contesto nei servizi per l'infanzia. Riflessioni ed esperienze, Bergamo: Edizioni Junior.

Bronfenbrenner U. (1979). Ecologia dello sviluppo umano. Trad. it. Bologna: Il Mulino, 1986.

Catarsi E. (2010). Coordinamento pedagogico e servizi per l'infanzia. Hoepli.

Dahlberg, G., Moss, P., \& Pence, A. (1999). Beyond quality in early childhood education and care: postmodern perspectives, (1. Ed.), London: Falmer Press.

Dewey, J. (1933). Come pensiamo. Trad. it. Firenze: la Nuova Italia, 1961.

European Comission. (2014). Proposal for key principles of a Quality Framework for Early Childhood Education and Care. Quality Framework. Version 2. (http://ec.europa.eu/education/policy/strategic-framework/archive/documents/ecec-quality-framework_en.pdf).

Freire, P. (1970). La pedagogia degli oppressi. Trad. it. Milano, Mondadori. Nuova edizione: Torino: EGA, 2002.

Gariboldi, A. (2015). Co-costruire la valutazione: le linee guida sulla valutazione della qualità in Regione Emilia Romagna. In: Bondioli A., Savio D. (a cura di). La valutazione di contesto nei servizi per l'infanzia. Riflessioni ed esperienze, Bergamo: Edizioni Junior, p. 167-175.

Montalbetti, K. (2005). La pratica riflessiva come ricerca educativa dell'insegnante. Milano: Vita e Pansiero.

Moss, P., Dahlberg, G. (2008). Beyond quality in early childhood education and carelanguages of evaluation. New Zealand Journal of Teacher's Work, 5 (1), p. 3-12.

Musatti, T., Mayer, S. (a cura di). (2003). Il coordinamento nei servizi educativi per l'infanzia. Una funzione emergente in Italia e in Europa, Azzano San Paolo, Junior. 
Salzberger-Wittenberg, I., Henry-Polacco, G., Osborne, E. (1983). The emotional experience of learning and teaching, London: Routledge and Kegan Paul.

Savio, D. (2013). La valutazione come promozione dall'interno. Reladei, 2 (2), Julio 2013, p. 69-86.

Savio, D. (2011). Il gioco e l'identità educativa del nido d'infanzia Un percorso di valutazione formativa partecipata nei nidi di Modena, Bergamo: Edizioni Junior.

Schön, D. (1983). Il professionista riflessivo, Bari: Edizioni Dedalo, 1993.

Silva, C. (2015). Il ruolo del coordinatore pedagogigo nella promozione dell'educazione e cura nella prima infanzia. Ensayos, Revista de la Facultad de Educación de Albacete, Italia, 30 (2), p. 205-212.

Texto recebido em 03 de novembro de 2016.

Texto aprovado em 13 de abril de 2017. 